\title{
TOKAMAK PLASMAS: A PARADIGM FOR CORONAL EQUILIBRIUM AND DISEQUILIBRIUM
}

\author{
Richard D. Petrasso \\ Plasma Fusion Center, MIT, Cambridge, MA 02139
}

\begin{abstract}
Tokamaks operate over a wide parameter space, allowing access to plasma conditions relevant to astrophysical plasmas. For high electron density discharges, for example, the central electron density and temperature are $\sim 3 \times$ $10^{14} \mathrm{~cm}^{-3}$ and $\sim 1.5 \mathrm{keV}$, and the central plasma region is in coronal equilibrium. Towards the edge of the plasma, however, many ion species will be far out of coronal equilibrium. A novel feature of the edge region is the seemingly contradictory property that it is, simultaneously, both a strongly recombining and a strongly ionizing plasma. Recent tokamak observations of strongly recombining plasmas also show that the $G$ parameter (the ratio of forbidden plus intercombination to resonance lines) is larger by a factor of 3 than the ratio of statistical weights of the tripletsinglet series. Such observations can be of direct consequence to the interpretation of non-equilibrium astrophysical plasmas.
\end{abstract}

There are several features of tokamak plasmas relevant to astrophysical ones. Primarily this is due to the wide variety of plasma conditions that can be readily accessed (Artsimovich 1972; Nuclear Fusion $\underline{25}$ 1985). For example, high temperature $\left(T_{e} \sim 3 \mathrm{keV} \simeq 35 \times 10^{6}{ }^{\circ} \mathrm{K}\right)$ plasmas are routinely obtained by running high field (8-10T), high current $(\sim 400 \mathrm{kA})$, and low electron density $\left(\leq 5 \times 10^{13} \mathrm{~cm}^{-3}\right)$ discharges (Parker et al. 1985). At these high temperatures, impurities such as titanium injected into a background plasma of hydrogen, will, upon reaching the plasma center, be ionized down to helium-like ions $\left(\mathrm{Ti}^{+20}\right)$. Of course, near the plasma edge where $T_{e} \sim 30 \mathrm{eV}$, only low-charge states of $\mathrm{Ti}$ will dominate. Even here, however, there will reside a small fraction of highly ionized titanium ions that have rapidly transported themselves from the center. Such highly stripped ions are - by orders of magnitude - out of coronal equilibrium, i.e. the edge plasma is strongly recombining. What is novel, however, is that the same physical region of the plasma is simultaneously a strongly ionizing one too! The reason for this is that a small fraction of slightly stripped $\mathrm{Ti}$ ions pierce,through the edge region in the direction of increasing temperature and, on the basis of the local electron temperature, possess too many bound electrons, i.e. they are underionized. It is through such rapid 
transport processes that the same physical location will have both under- and overionized ions, in steady state, but far out of coronal equilibrium. Superficially this situation appears unique in comparison to non-equilibrium astrophysical plasmas, which are often characterized as being either ionizing (Canizares et al. 1983) such as shock or flare-heated plasmas - or recombining, but not both. However, there may well be analogous ionizing-recombining (I-R) regions in astrophysical settings. One that suggests itself is the transition zone where the temperature gradient is extremely large: If local ion transit times through this zone are sufficiently small compared to characteristic ionization and recombination times, then it will be an I-R plasma. Another possible candidate is the boundary, or high-temperature gradient region, of a flare. Even if such I-R regions do exist astrophysically, one is still faced with the rather formidable task of confirming this observationally.

In order to make these comments about (non)equilibrium tokamak conditions more quantitative, it is useful to examine germane characteristics of a tokamak, such as MIT's Alcator-C (Parker et al. 1985). A tokamak is a torus-shaped structure with toroidal magnetic flux surfaces containing the plasma. Fig. 1a shows a crosssectional cut through the small-radius portion of the torus; as depicted, the coronal plasma (CP) occupies the central region and the ionizing-recombining plasma (I$\mathrm{RP}$ ) the outer annulus. (Of course there must be a third transition region between the two, but we will ignore such details here.) Directly below Fig. 1a is the electron temperature and density profile that characterizes local values of these parameters along the horizontal "cut" in Fig. 1a. Both the temperature and density are centrally peaked ( $1500 \mathrm{eV}$ and $4 \times 10^{14} \mathrm{~cm}^{-3}$ ) and monotonically decrease towards the plasma edge (about $16 \mathrm{~cm}$ from the plasma center). As shown, their exact functional dependence is very different, the electron temperature being more peaked. These profiles are important since they are needed to calculate the characteristic atomic times for ionization and recombination, i.e. $\tau_{A} \simeq 1 /\left[n_{e}(\sigma \nu)_{A}\right]$, where $(\sigma \nu)_{A}$ is the temperature dependent ionization or recombination rate coefficient appropriate for a given ion species. Fig. $1 \mathrm{c}$ shows the resulting spatial profiles of the atomic $\left(\tau_{A}\right)$ and ion transit or confinement time, $\tau_{t}$. $\left(\tau_{t}\right.$ is a semi-local function that is again evaluated along the horizontal cut of Fig. 1a.) The essential feature is that in the $\mathrm{CP}$ region, the plasma confinement time is longer than the atomic relaxation time, i.e. $\tau_{A} \leq \tau_{t}$. In contrast, for the I-R region the opposite is true, i.e., $\tau_{t} \leq \tau_{A}$.

Fig. 2 is a specific illustration of measured silicon ion profiles (solid curves) in the CP region, along with the corresponding coronal predictions (Petrasso et al. 1982). For this plasma discharge in the Alcator-C tokamak, a small amount of $\mathrm{Si}$ was injected into a background plasma of deuterium. Of importance to our considerations here is the fact that $\tau_{A} \sim 2 \mathrm{~ms}$ for highly stripped $\mathrm{Si}$, while, for the same ions, $\tau_{t} \sim 20 \mathrm{~ms}$. Within experimental error, deviations of the measured profiles from coronal equilibrium predictions were undetectable.

In contradistinction to the central coronal plasma, the edge plasma is strongly 
1 a).

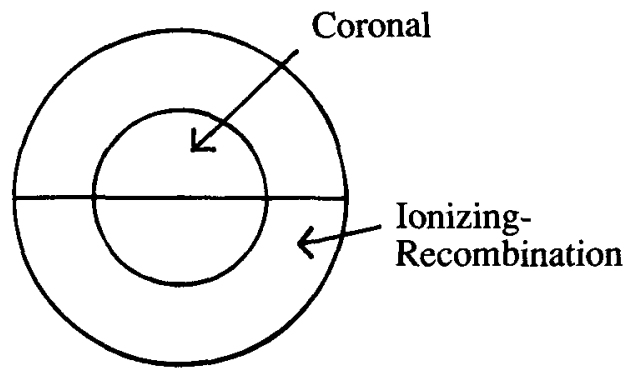

$1 \mathrm{~b})$.

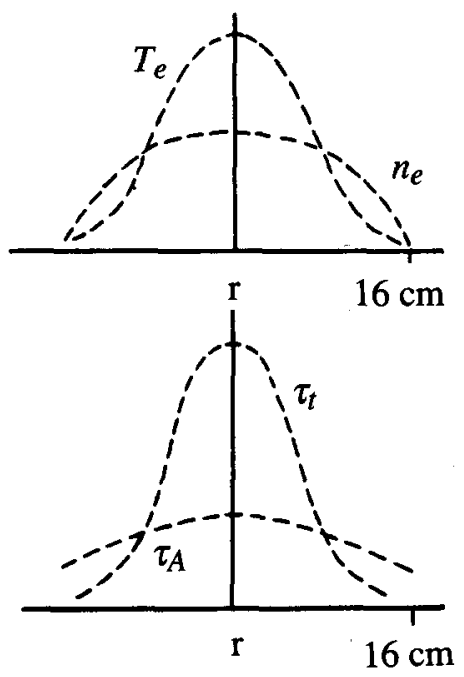

Fig. 1a.-A cross-sectional cut through the small radius portion of a tokamak plasma. From plasma center to very edge is about $16 \mathrm{~cm}$. For high-electron density discharges, the central plasma is in coronal equilibrium. The outer annulus, or "edge" region, is simultaneously both an ionizing and recombining plasma.

Fig. 1b.-The electron temperature $\left(T_{e}\right)$ and density $\left(n_{e}\right)$ profiles along the horizontal cut of Fig. 1a. In this instance, the peak values of $T_{\mathrm{e}}$ and $n_{\mathrm{e}}$ are about $1.5 \mathrm{keV}$ and $4 \times 10^{14} \mathrm{~cm}^{-3}$. The electron density profile is usually much broader than the temperature profile. The distance between the plasma center and outer edge is about $16 \mathrm{~cm}$ (based on Greenwald et al. 1984).

Fig. 1c.-A qualitative picture of the characteristic atomic times for ionization or recombination $\left(\tau_{A}\right)$, and the ion transit or confinement time $\left(\tau_{t}\right)$. The values are estimated along the horizontal cut of Fig. la. In the coronal region, $\tau_{A} \leq \tau_{t}$. In the ionizing-recombining region, $\tau_{A} \geq \tau_{t}$. At the plasma center, for example, $\tau_{A} \sim 2 m s$ for highly stripped $\mathrm{Si}$ while, in contrast, $\tau_{t} \sim 20 \mathrm{~ms}$ for the same ions. 

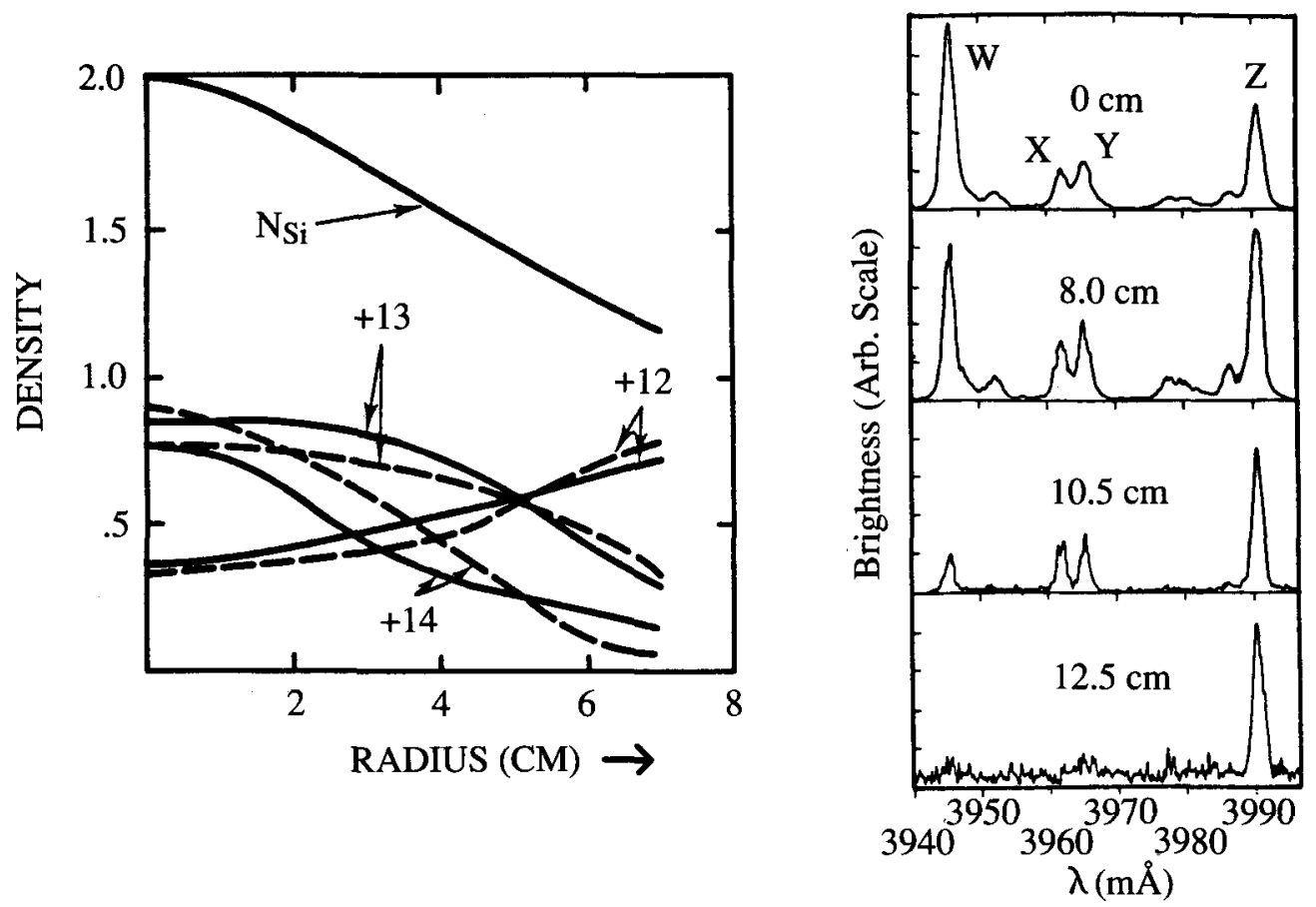

Fig. 2. Comparison between measured charged state Si profiles and the predictions of a coronal equilibrium model. Solid curves: Observed fully stripped $(+14), H-l i k e(+13)$, and He-like (+12) Si ion density profiles and their sum $\left(\mathrm{N}_{s} i\right)$. The central electron density and temperature are $3.4 \times 10^{14} \mathrm{~cm}^{-3}$ and $1.3 \mathrm{keV}$; the corresponding atomic relaxation times $\left(\tau_{A}\right)$ for highly stripped $\mathrm{Si}$ are about $2 \mathrm{~ms}$. In contrast, the transit or confinement time for these ions, $\tau_{t}$, is about $20 \mathrm{~ms}$ (from Petrasso et al. 1982).

Fig. 3. Spectra of helium-like argon at four different radial locations $(0$ through $12.5 \mathrm{~cm})$, showing the resonance $(\mathrm{W})$, intercombination $(\mathrm{X}$ and $\mathrm{Y}$ ), and forbidden $(\mathrm{Z})$ lines. (The vertical scales are a-bitrary.) Local electron temperatures at the four locations are 1650 , 550,250 and $100 \mathrm{eV}$. (For these Alcator-C plasma discharges, the plasma minor radius was $12.5 \mathrm{~cm}$.) Particularly intriguing - and still quantitatively unexplained - is the data at 12.5 $\mathrm{cm}$ which show the forbidden (Z) line to be completely dominant. The corresponding $\mathrm{G}$ value $[G=(X+Y+Z) / W]$ is $\geq 10$, which is much larger than that expected simply on the basis of the statistical weights of the triplet-singlet series (i.e. $G=3$ ) (from Rice et al. 1987). 
out of coronal equilibrium. John Rice and coworkers observed helium-like argon $\left(\mathrm{Ar}^{+16}\right)$ in the I-R region of the Alcator-C tokamak, and Fig. 3 shows a radial scan of the plasma with a crystal spectrometer which resolves the $\mathrm{W}$ (resonance), $\mathrm{X}$ and $Y$ (intercombination), and $Z$ (forbidden) lines of this ion (Rice et al. 1987). The G parameter $[\mathrm{G}=(\mathrm{Z}+\mathrm{X}+\mathrm{Y}) / \mathrm{W}]$, widely used in astrophysics as a plasma diagnostic (Jordan and Veck, 1982; Canizares et al. 1983; Canizares 1988; Mason 1988), is determined from these measurements. For the observations very near the plasma edge (the $12.5 \mathrm{~cm}$ case of Fig. 3), the spectrum is totally dominated by the forbidden line. In fact, the corresponding $G$ value is about 10 or greater. In analogous tokamak experiments at the Princeton Plasma Laboratory, but using helium-like titanium $\left(\mathrm{Ti}^{+20}\right)$, a $\mathrm{G}$ value of about 10 was also obtained (Bitter et al. 1985). In contrast, for strongly recombining plasmas such as these, it is commonly believed that the $\mathrm{G}$ value will asymptote to a value of order 3 , simply the ratio of statistical weights of the triplet-singlet series. Thus the mechanism by which the $2{ }^{3} \mathrm{~S}$ level, which corresponds to the upper state of the forbidden transition, becomes so dominantly populated is not, at present, quantitatively understood. It has been suggested, however, that the combination of charge-exchange recombination plus preferential cascading into $2{ }^{3} \mathrm{~S}$ state might resolve this enigma (Kallne et al. 1984). Therefore, such laboratory measurements as these could bear directly on the interpretation of plasma parameters in non-equilibrium astrophysical plasmas.

In summary, high density tokamak plasmas can be qualitatively divided into two regions, a central coronal region and an edge, ionizing-recombining one. In the coronal region, the atomic times for ionization and recombination are smaller than the characteristic ion confinement or transit times. In the ionizing-recombining region, the opposite prevails. In addition, because of the control that can be exercised over plasma conditions and impurities, a number of problems of astrophysical interest can be conveniently addressed in tokamak plasmas, and commonly used astrophysical diagnostics (e.g. the G parameter) can be subject to laboratory verification.

\section{ACKNOWLEDGMENTS}

For materials used in this presentation, I thank Chi-kang Li, Robert Granetz, John Rice and Kevin Wenzel. I gratefully acknowledge many helpful comments from Xing Chen and Dieter Sigmar. This work was supported by DOE contract, DE-AC02-78ET51013

\section{REFERENCES}

Artsimovich, L.A. 1972, Nuclear Fusion 6, 215.

Bitter, M., Hill, K.W., Zarnstorff, S., von Goeler, S., Hulse, R., Johnson, L.C., Sauthoff, N.R., Sesnic, S., and Young, K.M. 1985, Phys.Rev.A 32(5), 3011. 
Canizares, C.R., Winkler, P.F., Markert, T.H., and Berg, C. 1983, in Supernova Remnants and their X-ray Emission, J. Danziger and P. Gorenstein (eds.), IAU., 205.

Canizares, C.R. 1988, IAU Colloquium 115 (this conference), P. Gorenstein and M. Zombeck (eds).

Greenwald, M., Gwinn, D., Milora, S., Parker, J., Parker, R., Wolfe, S., Besen, B., Camacho, F., Fairfax, S., Fiore, C., Foord, M., Gandy, R., Gomez, C., Granetz, R., LaBombard, B., Lipschultz, B., Lloyd, B., Marmar, E., McCool, S., Pappas, D., Petrasso, R., Pribyl, P., Rice, J., Schuresko, D., Takase, Y., Terry, J., and Watterson, R. 1984, Phys.Rev.Lett. 53(4) 352.

Kallne, E., Kallne, J., Dalgarno, A., Marmar, E.S., Rice, J.E., and Pradhan, A.K. 1984, Phys.Rev.Lett. 52, 2245.

Jordan, C. and Veck, N.J. 1982, Solar Phys. 78, 125.

Mason, H. 1988, IAU Colloquium 115 (this conference), P. Gorenstein and M. Zombeck (eds.).

Nuclear Fusion 25(9), 1985; this volume contains several articles on tokamak programs throughout the world.

Parker, R., Greenwald, M., Luckhardt, M., Marmar, E.S., Porkolab, M., and Wolfe, S.M. 1985, Nuclear Fusion 25(9), 1127; references therein.

Petrasso, R.D., Seguin, F.H., Loter, N.G., Marmar, E., and Rice, J. 1982, Phys.Rev.Lett. 49, 1826.

Rice, J.E., Marmar, E.S., Kallne, E., and Kallne, J. 1987, Phys.Rev. A 35(7), 3033; references therein.

DISCUSSION-R. Petrasso

D. Sigmar: Tokomak experiments show $3 \mathrm{X}$ larger than predictions of coronal equilibrium theory for the Li-like to He-like ion ratio [M. Bitter et al. 1985 (Figure 10)]. Can impurity transport or other possibilities account for it?

R. Petrasso: An error or inaccuracy in the atomic physics calculations could account for the difference. Several workers have suggested that the ionization rate of Li-like ions should be reduced by about $50 \%$ with respect to the Lotz value. It is important to resolve this issue since astrophysicists commonly use the $\mathrm{Li}$ - to He-like ratio as a diagnostic to discern the non-equilibrium ionization state of astrophysical plasmas (Jordan and Veck 1982; Canizares et al. 1983). 